\title{
Volume do Stent à Ultra-Sonografia Intracoronária como Preditor de Reestenose Angiográfica. Estudo em Pacientes com Alto Risco de Reestenose
}

\author{
Moysés de Oliveira Lima-Filho', Geraldo Luiz de Figueiredo', Cleide Marques Antloga', \\ Silvio Sergio Pontes Câmara ${ }^{1}$, Maria Cristina Foss-Freitas², Milton Cesar Foss², José Antonio Marin-Neto ${ }^{1}$
}

\section{RESUMO}

Introdução: Em populações não selecionadas, obstruções em vasos de fino calibre são mais susceptíveis à reestenose após intervenção coronária percutânea (ICP). Por isso, entre as características angiográficas preditoras do fenômeno, o diâmetro luminal de referência (D.L.R.) é particularmente relevante. Tais resultados são corroborados por ultra-sonografia intracoronária (USIC), evidenciando a influência das dimensões vasculares sobre a resposta clínica no processo da reestenose, independente da presença de outros preditores, nessas populações não selecionadas. O objetivo deste trabalho foi avaliar os preditores angiográficos e ultra-sonográficos intracoronários de reestenose, em pacientes em que o alto risco de reestenose foi especialmente caracterizado por busca ativa de diabete melito (DM) e de disglicidemia. Método: Setenta pacientes portadores de 77 lesões obstrutivas ateroscleróticas coronarianas foram submetidos a ICP com stent, com sucesso. Realizou-se controle angiográfico e com USIC do resultado do implante, imediatamente após o término do procedimento e 6 meses após. Procedeu-se à busca ativa de alterações do metabolismo da glicose mediante a realização de GTTo, em todos os casos sem diagnóstico prévio de DM. Resultados: Foram identificados $23(32,86 \%)$ indivíduos com DM e 16 (22,85\%) com intolerância à glicose. Pela análise bivariada, os parâmetros considerados preditores de reestenose angiográfica pelo critério binário foram: D.L.R. $\geq 2,82$ mensurado na condição controle 6 meses após ICP - RR=0,60 (0,15-0,81) IC 95\% ( $p=0,014)$,

\section{SUMMARY}

Stent Volume as an Intracoronary Ultrasonographic Predictor of Restenosis. A Study in Patients with High Risk of Restenosis

Background: In non-selected populations, coronary obstructions of small vessels are more likely to suffer restenosis after percutaneous interventions. Thus, the reference luminal diameter (RLD) is a particularly important angiographic parameter, which is inversely related to the restenosis rate. Additionally in non-selected patients, ultrasound (IVUS) studies have shown similar results regarding the influence of vascular dimensions as independent predictors of clinical restenosis. The aim of this study was to evaluate the angiographic and IVUS parameters as predictors of restenosis in patients submitted to $\mathrm{PCl}$ with stents in whom a high risk for restenosis was characterized in a prospective search for diabetes mellitus and dysglycemia. Methods: Seventy patients with 77 coronary artery obstructions were submitted to successful $\mathrm{PCl}$ with stents. Quantitative analysis of coronary angiograms and intracoronary ultrasonographic images were obtained immediately after and six months after the index procedure. An oral GTT test was performed in all patients without prior diagnosis of diabetes mellitus. Results: Twenty-three diabetics $(32.86 \%)$ and 16 patients with glucose intolerance $(22.85 \%)$ were identified. The angiographic and IVUS parameters considered predictors of restenosis through bivariate analysis were: $R L D \geq 2.82 \mathrm{~mm}$ six months after the procedure - RR=0.60

\footnotetext{
1 Unidade de Cardiologia Intervencionista, Divisão de Cardiologia, Departamento de Clínica Médica, Hospital das Clínicas, Faculdade de Medicina de Ribeirão Preto da Universidade de São Paulo.

2 Divisão de Endocrinologia e Metabologia, Departamento de Clínica Médica, Hospital das Clínicas, Faculdade de Medicina de Ribeirão Preto da Universidade de São Paulo.

Este trabalho de investigação compôs parte da Tese de Doutorado defendida junto à Faculdade de Medicina de Ribeirão Preto, USP, por Moysés de Oliveira Lima Filho, em 22 de setembro de 2003, e foi parcialmente subvencionado pela FAPESP, processo no 99/07143-2. Correspondência: Moysés de Oliveira Lima-Filho. Unidade de Cardiologia Intervencionista, Divisão de Cardiologia, Departamento de Clínica Médica, Hospital das Clínicas, Faculdade de Medicina de Ribeirão Preto, USP. Avenida Bandeirantes, 3900. Campus da USP em Ribeirão Preto - Ribeirão Preto, SP - CEP 14048-900.

Tel.: (16) 3602-2599 • E-mail: moyslima@usp.br

Recebido em: 12/03/2007 • Aceito em: 12/04/2007
} 
Lima-Filho MO, et al. Volume do Stent à Ultra-Sonografia Intracoronária como Preditor de Reestenose Angiográfica. Estudo em Pacientes com Alto Risco de Reestenose. Rev Bras Cardiol Invas 2007; 15(2): 125-133.

volume de stent $<119,8 \mathrm{~mm}^{3}$ ao USIC - RR=0,74 $(0,38-$ $0,89)$ IC $95 \%(p=0,0005)$ e área de stent $\leq 8,91 \mathrm{~mm}^{2}$ ao USIC - RR=0,66 (0,24-0,85) IC 95\% ( $p=0,006)$. A análise multivariada evidenciou que o único parâmetro preditor de reestenose foi volume de stent $<119,8 \mathrm{~mm}^{3}(\mathrm{p}=0,01)$. Conclusão: Com base nestes resultados, conclui-se que, em população de pacientes com alto risco para desenvolvimento de reestenose, o calibre do vaso esteve relacionado de forma inversa à taxa de reestenose, sendo o volume de stent pelo USIC $<119,8 \mathrm{~mm}^{3}$ considerado preditor independente de reestenose.

DESCRITORES: Angioplastia transluminal percutânea coronária. Reestenose coronária. Ultra-sonografia de intervenção. Diabetes mellitus.
A reestenose após intervenção coronária percutânea (ICP) é definida angiograficamente pela volta de lesão reduzindo em $\geq 50 \%$ o diâmetro luminal ${ }^{1}$. Esta definição binária, que divide a população de forma qualitativa em dois grandes grupos, mesmo tendo a linha de corte bem definida e estabelecida com exatidão por angiografia quantitativa, é criticável por não corresponder ao contexto clínico em proporção substancial dos casos. Essa restrição conceitual é obviada na prática clínica ${ }^{2}$, sendo a necessidade de nova revascularização para tratar a lesão índice o parâmetro de escolha comumente usado para traduzir o fenômeno da reestenose coronária $(\mathrm{RC})^{3-5}$. Não obstante tal fato, o estudo dos aspectos relacionados ao procedimento, como preditores de RC, é fundamental para melhor entendimento dos mecanismos fisiopatológicos envolvidos.

Desde a era precedente ao uso das endopróteses coronarianas, verificou-se que vasos de fino calibre são mais susceptíveis à RC angiográfica pelo critério binário. Esse conceito genérico apóia-se em que, entre as características angiográficas preditoras do fenômeno, o diâmetro luminal de referência (D.L.R.) do vaso dilatado é particularmente relevante. Nos estudos da fase inicial da ICP, também se mostravam preditores significantes de RC o diâmetro luminal mínimo (D.L.M.) e a porcentagem de estenose ao término do procedimento terapêutico ${ }^{6}$. Entretanto, mesmo com o uso atual disseminado dos stents e sendo a RC após implante dos mesmos dependente, essencialmente, de hiperproliferação celular, com neoformação miointimal' ${ }^{7}$, D.L.R. continuou a ser caracterizado como preditor independente de $\mathrm{RC}^{8,9}$. Esta verificação, primordialmente derivada de estudos angiográficos, confirmou-se em estudos com ultra-sonografia intracoronária (USIC), mostrando-se que o calibre do vaso tratado interfere nitidamente na probabilidade de $\mathrm{RC}^{10,11}$. Em contraste, apenas o diabete melito (DM), e, possivelmente, a instabilidade do quadro clínico, são considerados atualmente fatores clínicos preditores de reestenose $\mathrm{e}^{12-14}$.

Embora em pacientes diabéticos as características
(0.15 - 0.81) 95\% Cl ( $\mathrm{p}=0.014)$, Stent Volume $<119.8 \mathrm{~mm}^{3}-$ $\mathrm{RR}=0.74(0.38-0.89) 95 \% \mathrm{Cl}(\mathrm{p}=0.0005)$ and Stent Area $\leq 8.91 \mathrm{~mm}^{2}-\mathrm{RR}=0.66(0.24-0.85) 95 \% \mathrm{Cl}(\mathrm{p}=0.006)$. By logistic regression multivariate analysis, stent volume $\mathrm{mm}^{3}$ $<119.8$ was considered the only independent predictor of coronary restenosis $(p=0.01)$. Conclusions: In this population with high risk for coronary restenosis, vessel dimensions were inversely related to its occurrence. A stent volume $<119.8 \mathrm{~mm}^{3}$ measured by IVUS was the only independent predictor of restenosis after $\mathrm{PCI}$.

DESCRIPTORS: Angioplasty, transluminal, percutaneous coronary. Coronary restenosis. Ultrasonography, interventional. Diabetes mellitus.

angiográficas e ultra-sonográficas relacionadas com o calibre dos vasos e das lesões tratadas também sejam consideradas preditoras ${ }^{15,16}$, há controvérsias, pelo fato de grande parte dos estudos ser anterior ao conceito de DM em vigor atualmente. Além disso, deve-se considerar que o DM é relativamente subdiagnosticado na população geral, bem como na de coronariopatas tratados com ICP. Assim, mesmo nos grandes estudos, a percepção do DM costuma ser baseada em diagnóstico estabelecido de forma não sistematizada, antes do procedimento, e nem sempre sendo realizada busca ativa de novos casos com a doença. Finalmente, são muito limitadas as investigações relatadas de verificação dos fatores angiográficos e ultra-sonográficos preditores de RC em pacientes com outras formas de disglicidemia ${ }^{17}$, como a intolerância à glicose e a glicemia de jejum alterada, estados considerados pré-diabéticos, nos quais ocorre um grau aumentado de resistência insulínica comparativamente à população sem alterações do metabolismo da glicose.

\section{OBJETIVO}

Este estudo teve como objetivo primário avaliar os preditores angiográficos e ultra-sonográficos de RC após ICP com stents em grupo de pacientes na qual foi realizada busca ativa de distúrbios do metabolismo da glicose, de forma a caracterizar-se mais rigorosamente população com risco elevado de desenvolver RC.

\section{MÉTODO}

\section{Características clínicas}

De fevereiro de 2001 a agosto de 2002, foram incluídos, prospectiva e consecutivamente, desde que atendessem aos critérios de inclusão e de exclusão pré-fixados, 76 pacientes portadores de 87 lesões coronárias ateroscleróticas obstrutivas tratadas por ICP com implante de stents. Em todos os casos, nesta fase, realizou-se angiografia quantitativa e USIC. 
Lima-Filho MO, et al. Volume do Stent à Ultra-Sonografia Intracoronária como Preditor de Reestenose Angiográfica. Estudo em Pacientes com Alto Risco de Reestenose. Rev Bras Cardiol Invas 2007; 15(2): 125-133.

Desse grupo inicial, em 70 indivíduos, portadores de 77 lesões, conseguiu-se realizar reestudo cineangiocoronariográfico, após seis meses, complementado com repetição da USIC. Todos os pacientes, antes do primeiro procedimento, assinaram termo de consentimento após informação circunstanciada, e o estudo foi aprovado pelo Comitê de Ética em Pesquisa Médica do Hospital das Clínicas da Faculdade de Medicina de Ribeirão Preto da Universidade de São Paulo.

\section{Critérios de inclusão}

Pacientes portadores de coronariopatia obstrutiva, maiores de 18 anos de idade, sem limite superior de idade, de ambos os gêneros, em pleno exercício de suas faculdades mentais, que pudessem entender o significado da pesquisa e concordar com o termo de consentimento; presença de isquemia miocárdica detectada clínica e/ou laboratorialmente e de lesão obstrutiva coronária passível de tratamento por angioplastia com implante de stent, nas seguintes condições: $\geq 70 \%$ de obstrução em vasos com D.L.R. acima de $2,5 \mathrm{~mm}$ de diâmetro, sendo ambas as avaliações pela estimativa visual coronariográfica. A limitação em relação ao D.L.R. visa a garantir maior segurança na passagem do cateter de ultra-sonografia intravascular, assim como no implante dos stents.

\section{Critérios de exclusão}

Lesões localizadas em tronco de coronária esquerda não protegido por cirurgia de revascularização miocárdica prévia; lesões com imagem angiográfica sugestiva de trombo; lesões em pontes de veia safena com imagem angiográfica de degeneração parietal; lesões obstruindo totalmente a luz do vaso; mulheres em período gestacional; pacientes em choque cardiogênico; pacientes em fase aguda de infarto do miocárdio (primeiras 72 horas).

Como visto na Tabela 1, estes indivíduos eram predominantemente do gênero masculino, com média de idade de 62 anos. Houve nítido predomínio de pacientes nos quais os procedimentos terapêuticos foram realizados após fase de estabilização clínica por terem apresentado síndromes isquêmicas miocárdicas instáveis. A maioria dos pacientes apresentava função sistólica do VE preservada (78,57\%). O acometimento coronário era univascular em 45,71\%, bivascular em $41,43 \%$ e trivascular em $12,86 \%$ da amostra.

Visando a estabelecer diagnóstico de alterações do metabolismo da glicose (diglicidemia), todos os pacientes realizaram teste oral de tolerância à glicose (GTTo), duas semanas após o procedimento, e foram classificados de acordo com esses resultados, em adição à glicemia de jejum, nos seguintes grupos: a) diabéticos; b) intolerantes e c) normais, de acordo com os critérios estipulados pela Organização Mundial de Saúde $(\mathrm{OMS})^{18}$. O grupo de pacientes diabéticos foi composto por $23(32,86 \%)$ indivíduos, o de intolerância à glicose por $16(22,85 \%)$ e o de normoglicemia por 31
$(44,28 \%)$. Cabe ressaltar, portanto, que mais da metade da amostra estudada apresenta distúrbio do metabolismo da glicose.

\section{Aspectos angiográficos qualitativos basais}

Foram tratadas 77 lesões obstrutivas de localização predominantemente proximal (51,95\%), mas apenas uma lesão era ostial em coronária descendente anterior. Quase $60 \%$ das lesões eram complexas, classificadas como B2 ou C. O vaso tratado foi a DA em $46,75 \%$ das vezes. Apenas uma lesão era reestenótica, tendo sido previamente tratada por angioplastia com balão sem stent (Tabela 2).

Foram utilizados oito diferentes tipos de stents, os disponíveis no serviço durante o período. Não houve definição protocolar quanto ao tipo de stent a ser utilizado, ficando este a critério do operador, assim como quanto à pressão final de insuflação durante implante, que foi em média \pm DP, 15,1 \pm 1,98 atm.

Todos os pacientes receberam antiagregação plaquetária combinada com ácido acetilsalicílico (AAS) e ticlopidina ou clopidogrel, por período mínimo de 15 dias após o procedimento. Depois, todos continuaram em uso de AAS indefinidamente. Nenhum paciente

TABELA 1

Aspectos clínicos gerais

\begin{tabular}{|c|c|c|}
\hline & $\mathbf{N}$ & $\%$ \\
\hline Total & 70 & 100,0 \\
\hline Idade* (anos) & \multicolumn{2}{|c|}{$62\left(47 a_{-} 85\right)$} \\
\hline Índice de massa corporal $(\mathrm{IMC})^{+}$ & \multicolumn{2}{|c|}{$26,55 \pm 4,27$} \\
\hline Gênero masculino & 54 & 77,14 \\
\hline Assintomáticos & 8 & 11,43 \\
\hline Equiv. anginoso & 1 & 1,43 \\
\hline Angina estável & 15 & 21,43 \\
\hline Angina instável & 28 & 40,0 \\
\hline IAM sem supra de ST & 1 & 1,43 \\
\hline IAM com supra de ST & 17 & 24,29 \\
\hline HAS & 57 & 81,43 \\
\hline Obesidade & 16 & 22,86 \\
\hline Dislipidemia & 40 & 57,14 \\
\hline Hist. Familiar & 30 & 42,86 \\
\hline Diabetes Mellitus & 23 & 32,86 \\
\hline Intolerantes à Glicose & 16 & 22,86 \\
\hline Tabagismo & 21 & 30,0 \\
\hline FEVE Normal & 55 & 78,57 \\
\hline Uniarterial & 32 & 45,71 \\
\hline Biarterial & 29 & 41,43 \\
\hline Triarterial & 9 & 12,86 \\
\hline
\end{tabular}

* Idade expressa em média e extremos

+ IMC expresso em média e desvio-padrão

$\mathrm{FEVE}=$ fração de ejeção do ventrículo esquerdo 
Lima-Filho MO, et al. Volume do Stent à Ultra-Sonografia Intracoronária como Preditor de Reestenose Angiográfica. Estudo em Pacientes com Alto Risco de Reestenose. Rev Bras Cardiol Invas 2007; 15(2): 125-133.

TABELA 2

Características angiográficas basais

\begin{tabular}{lrc}
\hline & N & $\%$ \\
\hline Total & 77 & 100,0 \\
Óstio & 1 & 1,30 \\
Segmento proximal & 40 & 51,95 \\
Segmento médio & 32 & 41,56 \\
Segmento distal & 4 & 5,19 \\
Tipo A & 9 & 11,69 \\
Tipo B1 & 23 & 29,87 \\
Tipo B2 & 44 & 57,14 \\
Tipo C1 & 1 & 1,30 \\
CD & 27 & 35,07 \\
CX & 9 & 11,69 \\
DA & 36 & 46,75 \\
Diagonalis & 1 & 1,30 \\
Marginal & 4 & 5,19 \\
$<10$ mm & 36 & 46,75 \\
$>$ 10 e < 20 mm & 40 & 51,95 \\
$>$ 20 mm & 1 & 1,30 \\
Excentricidade & 49 & 63,6 \\
Tortuosidade & 4 & 5,19 \\
Angulação & 12 & 15,58 \\
Calcificação & 15 & 19,48 \\
Lesão reestenótica & 1 & 1,30 \\
Bifurcação & 6 & 7,79 \\
Bordas irregulares & 3 & 3,90 \\
\hline
\end{tabular}

recebeu inibidores de glicoproteína Ilb/IIla. Outros medicamentos foram utilizados a critério clínico, tendo sido recomendado o uso de vasodilatador coronário (nitratos e/ou bloqueadores de canais de cálcio) no período hospitalar.

\section{Análise angiográfica quantitativa}

Realizada off-line com sistema CAAS-II (Pie Medical Imaging B.V., Maastricht, Holanda), previamente validado $^{19-22}$. Todos os angiogramas foram realizados após injeção intracoronária de 0,2 mg de nitroglicerina. Os cálculos e medidas apresentados derivam da análise de dois planos ortogonais em cada uma das três condições do estudo (antes e depois da ICP e controle tardio aos seis meses). Sempre foram avaliados os quadros cinecoronariográficos durante a diástole, nos quais a obstrução mostrava-se mais intensa, e evitando-se a sobreposição de vasos. Para cada condição, utilizou-se o menor D.L.M., e a média do D.L.R. entre as duas projeções, sendo os demais cálculos obtidos a partir destes dois parâmetros. Foram observadas, cuidadosamente, as projeções realizadas na situação pré-procedimento, a fim de que as mesmas pudessem ser repetidas nas situações pós-procedimento e controle tardio após seis meses.

\section{Ultra-sonografia intracoronária}

O estudo com USIC foi realizado em todos os pacientes, imediatamente após o procedimento e no final do período de controle após seis meses.

Para captação das imagens ultra-sonográficas foram utilizados cateteres com transdutores de 30 ou 40 $\mathrm{MHz}$, acoplados aos sistemas Clear View ${ }^{\mathrm{TM}}$ ou Galaxy ${ }^{\mathrm{TM}}$ (Boston Scientific, Maple Grove, Minnesota), utilizados para subseqüente visualização das imagens gravadas e realização de análise quantitativa. Para aquisição dinâmica das imagens, o cateter foi adaptado a sistema de recuo escalonado progressivo, com velocidade fixa de $0,5 \mathrm{~mm} / \mathrm{seg}$. Imediatamente antes desta aquisição, infundia-se $0,2 \mathrm{mg}$ de nitroglicerina intracoronária através da via de infusão do cateter de USIC. É importante salientar-se que o resultado do procedimento terapêutico não foi em geral guiado, mas sim documentado por USIC. Entretanto, em 5 pacientes, diante da análise ultra-sonográfica, optou-se por complementação do procedimento com nova dilatação da endoprótese por balão, e tornando-se a passar o cateter ultra-sonográfico após esta complementação, com estes resultados considerados para análise.

Pela análise ultra-sonográfica quantitativa foram observados os seguintes parâmetros, de acordo com as várias fases dos estudos, em cortes transversais seriados do vaso a cada milímetro: Área Luminal em $\mathrm{mm}^{2}$ (A.L.); Área delimitada pelo Stent em $\mathrm{mm}^{2}$ (A.S.); Área de Hiperplasia Neointimal em $\mathrm{mm}^{2}$ (A.H.N.) = A.S. - A.L.; Razão entre Área de Hiperplasia Neointimal e Área delimitada pelo Stent (A.H.N./A.S.). A partir dessas medidas de área e com base na fórmula de Simpson ${ }^{23}$, na qual a média das áreas planimetradas a cada milímetro é multiplicada pelo comprimento da extensão medida, foram calculados os seguintes dados volumétricos após o implante do stent: Volume Luminal em $\mathrm{mm}^{3}$ (V.L.); Volume delimitado pelo Stent $\mathrm{em}^{\mathrm{m}} \mathrm{m}^{3}$ (V.S.); Volume de Hiperplasia Neointimal em $\mathrm{mm}^{3}$ (V.H.N.); Relação entre Volume de Hiperplasia Neointimal e Volume delimitado pelo Stent (V.H.N./V.S.).

\section{Análise estatística}

As variáveis qualitativas foram avaliadas a partir dos testes de Chi-quadrado e exato de Fischer. As variáveis quantitativas foram usualmente avaliadas a partir do teste de Kruskal-Wallis ou pelo teste " $\mathrm{t}$ " de Student, quando o parâmetro analisado teve distribuição normal na amostra investigada. Foram realizadas regressão linear e correlação por "ranks" de Spearman, e quando o parâmetro a ser medido apresentava distribuição gaussiana, foi realizado método de correlação de Pearson, assim como análise de variância para determinação dos prováveis parâmetros clínicos e laboratoriais associados ao procedimento terapêutico que poderiam estar relacionados com maior grau de hiperproliferação neointimal. A avaliação dos preditores de reestenose foi feita a partir de análise bi e multivariada 
Lima-Filho MO, et al. Volume do Stent à Ultra-Sonografia Intracoronária como Preditor de Reestenose Angiográfica. Estudo em Pacientes com Alto Risco de Reestenose. Rev Bras Cardiol Invas 2007; 15(2): 125-133.

por regressão logística, utilizando-se o critério binário a partir do valor da mediana do parâmetro analisado.

\section{RESULTADOS}

O D.L.R. foi de 2,92 $\pm 0,49 \mathrm{~mm}$, na condição basal (pré-procedimento), e de $3,18 \pm 0,44$, no pós-procedimento imediato, valores não discrimináveis estatisticamente. O D.L.M. basal foi de 0,82 $\pm 0,29 \mathrm{~mm}$ e imediatamente após o tratamento alcançou 2,68 $\pm 0,38 \mathrm{~mm}$, um ganho agudo de 1,87 $\pm 0,43 \mathrm{~mm}$; o grau de estenose de $71,34 \pm 9,48 \%$, na condição basal, passou para $15,5 \pm 6,17 \%$, no pós-procedimento imediato. O comprimento da lesão na condição basal foi de 11,44 \pm $3,86 \mathrm{~mm}$.

Pela cinecoronariografia de controle tardio após 6 meses, a taxa de reestenose pelo critério binário foi de $31,17 \%$, ocorrendo em $12(44,4 \%)$ das 27 lesões tratadas em pacientes diabéticos, em $4(25,0 \%)$ das 16 lesões em pacientes intolerantes à glicose e em 8 $(22,8 \%)$ das 34 lesões tratadas em pacientes normoglicêmicos. Tais resultados evidenciam tendência a maior incidência de reestenose pelo critério binário, em pacientes diabéticos $(p=0,07)$ comparativamente aos demais grupos.

Analisando as características clínicas de acordo com a ocorrência ou não de reestenose, não se encontram diferenças significantes estatisticamente entre os dois grupos para cada aspecto avaliado, excetuandose a comparação síndrome coronária isquêmica aguda versus crônica. O tratamento de lesões em quadro clínico estável leva à redução de risco relativo de reestenose coronária, que assume o valor de 0,72 $(0,15-0,91) \quad I C=95 \%, p=0,009$.

\section{Preditores de reestenose por angiografia quantitativa}

Os preditores angiográficos quantitativos de reestenose pela análise bivariada foram os diâmetros luminais de referência aferidos em qualquer das três medidas efetuadas nas condições deste estudo. Um D.L.R. $\geq 2,93$ (mediana da amostra) na condição basal levou à redução do risco de reestenose: $R R=0,54(0,05-$ $0,78)$ IC $95 \%(p=0,048)$. Na condição após procedimento imediata, também pelo valor da mediana da amostra, um valor de D.L.R. $\geq 3,19$ levou à redução de risco de reestenose: $R R=0,62(0,19-0,82)$ IC $95 \%$ $(p=0,013)$. O mesmo ocorreu na condição controle de seis meses, pela qual o D.L.R. $\geq 2,82$ levou à redução de risco relativo de reestenose: $R R=0,60(0,15-0,81)$ IC $95 \% \quad(p=0,014)$.

O D.L.M., ao final do procedimento, não se mostrou preditor de reestenose pela análise bivariada, considerando-se valores inferiores e superiores da mediana da amostra geral, que foi igual a 2,64 mm ( $p=0,220)$. No entanto, ao considerar-se a comparação das médias do D.L.M., ao final do procedimento, entre as lesões reestenóticas versus não reestenóticas, houve tendência a menores diâmetros luminais mínimos estarem correlacionados com maior taxa de reestenose $(p=0,0688)$, como disposto na Tabela 3 .

As lesões reestenóticas, em média, apresentavam menor grau de estenose ao término do procedimento $(p=0,0295)$. Este resultado paradoxal provavelmente é devido à diferença de D.L.R. entre os dois grupos. Assim, pela análise bivariada da mediana da amostra (grau de estenose $>16,0 \%$ ), aqueles resultados contra-

TABELA 3

Análise angiográfica quantitativa nas lesões reestenóticas versus não reestenóticas

\begin{tabular}{lccc}
\hline & Com reestenose & Sem reestenose & p \\
\hline DLR (mm) - pré & $2,64 \pm 0,46$ & $3,03 \pm 0,45$ & 0,0008 \\
DLR (mm) - pós & $2,95 \pm 0,41$ & $3,29 \pm 0,39$ & 0,001 \\
DLR (mm) - controle 6 meses & $2,64 \pm 0,46$ & $2,96 \pm 0,51$ & 0,0102 \\
DLM (mm) - pré & $0,79 \pm 0,28$ & $0,83 \pm 0,29$ & 0,7126 \\
DLM (mm) - pós & $2,57 \pm 0,36$ & $2,74 \pm 0,38$ & 0,0688 \\
DLM (mm) - controle 6 meses & $0,91 \pm 0,39$ & $2,07 \pm 0,47$ & $<0,0001$ \\
Grau de estenose (\%) - pré & $69,68 \pm 8,66$ & $72,26 \pm 9,65$ & 0,235 \\
Grau de estenose (\%) - pós & $13,51 \pm 5,80$ & $16,35 \pm 5,86$ & 0,0295 \\
Grau de estenose (\%) - controle 6 meses & $65,6 \pm 12,8$ & $30,9 \pm 9,9$ & $<0,0001$ \\
Comprimento (mm) & $10,42 \pm 3,74$ & $11,98 \pm 3,96$ & 0,1089 \\
Ganho agudo (mm) & $1,91 \pm 0,43$ & $1,78 \pm 0,41$ & 0,2861 \\
Ganho agudo relativo (mm) & $0,63 \pm 0,14$ & $0,69 \pm 0,21$ & 0,1451 \\
Perda tardia (mm) & $0,67 \pm 0,44$ & $1,67 \pm 0,44$ & $<0,0001$ \\
Perda tardia relativa (mm) & $0,22 \pm 0,15$ & $0,65 \pm 0,20$ & $<0,0001$ \\
Índice de perda tardia & $0,35 \pm 0,23$ & $0,96 \pm 0,27$ & $<0001$ \\
Índice de perda tardia relativa & $0,12 \pm 0,08$ & $0,37 \pm 0,12$ & $<, 0001$ \\
\hline
\end{tabular}


Lima-Filho MO, et al. Volume do Stent à Ultra-Sonografia Intracoronária como Preditor de Reestenose Angiográfica. Estudo em Pacientes com Alto Risco de Reestenose. Rev Bras Cardiol Invas 2007; 15(2): 125-133.

ditórios não se repetiram, havendo tendência $(p=0,073)$ de graus mais intensos de estenose após o procedimento estarem relacionados com maior incidência de reestenose pelo critério binário angiográfico.

Conforme demonstrado na Tabela 3, o ganho agudo absoluto ou relativo do diâmetro luminal mínimo com o procedimento terapêutico não esteve relacionado com o fenômeno da reestenose. Isto se confirmou pela análise bivariada.

\section{Ultra-sonografia intracoronária: análise quantitativa}

Os resultados das análises quantitativas de área e volumétrica pela ultra-sonografia controle realizada após seis meses estão dispostos na Tabela 4. Por análise bivariada, a presença de volume de stent $<119,8 \mathrm{~mm}^{3}$ foi preditora de reestenose com base no critério binário angiográfico, sendo o risco relativo para volumes acima deste valor numérico igual a $0,74(0,38-0,89)$ IC 95\% com um valor de $p=0,0005$. O mesmo ocorreu em relação à presença de área de stent $\leq 8,91 \mathrm{~mm}^{2}$, pois para valores superiores aos da mediana registrouse RR de reestenose de 0,66 $(0,24-0,85)$ IC 95\%, com um valor de $p=0,006$. A Tabela 4 mostra que as diferenças entre as médias de volume de stent e de área de stent nas lesões reestenóticas versus não reestenóticas são altamente significativas.

As Figuras 1 e 2 mostram os gráficos de regressão linear entre os graus de estenose pela cinecoronariografia no controle de 6 meses e os volumes e áreas de stent pela USIC também dessa fase. Embora os baixos valores do coeficiente de correlação de Pearson, houve significância estatística, em ambos os casos.

\section{Análise multivariada dos preditores de reestenose}

Conforme exposto, pela análise bivariada, dentre todos os parâmetros angiográficos e ultra-sonográficos avaliados, somente o D.L.R., e a área e o volume de stent foram preditores de reestenose, como definido angiograficamente pelo critério binário.

No entanto, a análise multivariada por regressão

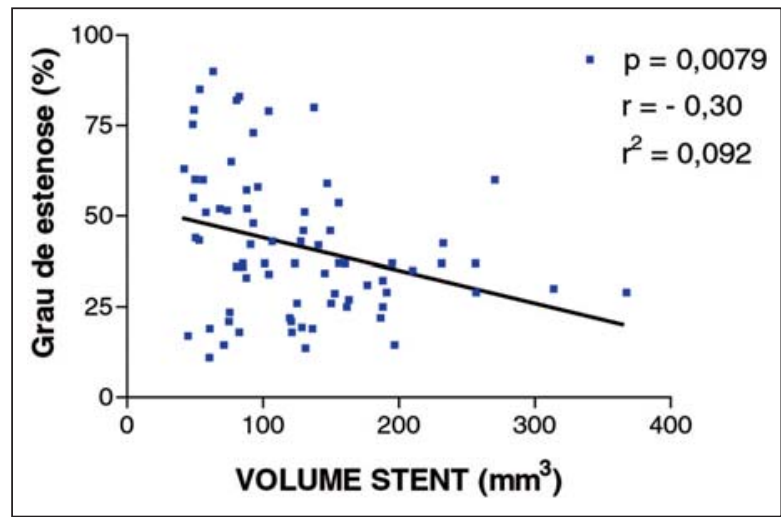

Figura 1 - Correlação entre grau de estenose pela angiografia e o volume de stent por USIC na fase de controle após 6 meses.

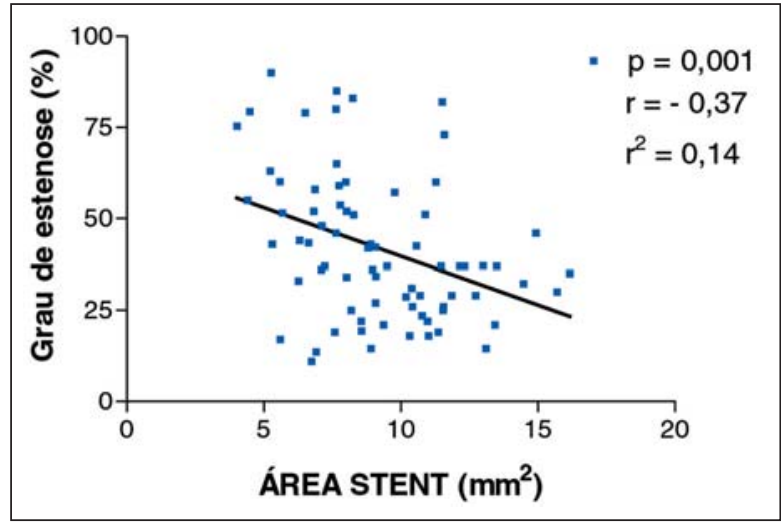

Figura 2 - Correlação entre grau de estenose pela angiografia e área de stent por USIC na fase de controle após 6 meses.

TABELA 4

Análise ultra-sonográfica quantitativa na fase de controle após seis meses nas lesões reestenóticas versus não reestenóticas

\begin{tabular}{lcrr} 
& Com reestenose & Sem reestenose & p \\
\hline Área da luz $\left(\mathrm{mm}^{2}\right)$ & $1,98 \pm 0,89$ & $5,37 \pm 2,23$ & $<0,0001$ \\
Área do stent $\left(\mathrm{mm}^{2}\right)$ & $7,53 \pm 2,23$ & $10,04 \pm 2,65$ & 0,0003 \\
A.H.N. $\left(\mathrm{mm}^{2}\right)$ & $5,54 \pm 2,02$ & $4,66 \pm 1,51$ & 0,0746 \\
A.H.N./área stent $(\%)$ & $72,0 \pm 10,0$ & $47,0 \pm 12,0$ & $<0,0001$ \\
Volume da luz $\left(\mathrm{mm}^{3}\right)$ & $40,11 \pm 32,14$ & $97,13 \pm 51,52$ & $<0,0001$ \\
Volume do stent $\left(\mathrm{mm}^{3}\right)$ & $90,03 \pm 50,49$ & $142,01 \pm 67,29$ & 0,0003 \\
V.H.N. $\left(\mathrm{mm}^{3}\right)$ & $49,92 \pm 25,54$ & $44,88 \pm 25,49$ & 0,3975 \\
V.H.N./volume do stent $(\%)$ & $57,0 \pm 15,0$ & $32,0 \pm 10,0$ & $<0,0001$ \\
\hline
\end{tabular}

A.H.N.= área de hiperplasia neointimal; V.H.N.= volume de hiperplasia neointimal 
Lima-Filho MO, et al. Volume do Stent à Ultra-Sonografia Intracoronária como Preditor de Reestenose Angiográfica. Estudo em Pacientes com Alto Risco de Reestenose. Rev Bras Cardiol Invas 2007; 15(2): 125-133.

logística evidenciou que o único parâmetro preditor de reestenose foi volume de stent $<119,8 \mathrm{~mm}^{3}(p=0,01)$ - Tabela 5.

\section{DISCUSSÃO}

O presente estudo demonstra que também em amostra populacional na qual o alto risco de reestenose é caracterizado por se constituir de mais de $50 \%$ de pacientes disglicêmicos, a taxa de RC é nítida e inversamente correlacionada ao calibre vascular, conforme documentado por análise angiográfica quantitativa e ultra-sonográfica. Enquanto os presentes resultados foram obtidos com aproximadamente 55\% de pacientes diabéticos ou intolerantes à glicose, a grande maioria dos estudos descreve amostras populacionais em que pacientes diabéticos correspondem a apenas 20-25\% da casuística. Essa incidência de distúrbios glicêmicos encontrada no atual estudo é congruente com publicação de Muhlestein et al. ${ }^{24}$, na qual anormalidades do metabolismo da glicose tiveram prevalência de 61\%, em pacientes tratados com ICP.

Entre os aspectos clínicos avaliados, apenas as síndromes isquêmicas miocárdicas instáveis (SIMIs) foram consideradas preditoras de reestenose pela análise bivariada. A presença de SIMIs como preditor de reestenose após implante de stents não é descrita na literatura, diversamente do que ocorria na era pré-stent ${ }^{25-28}$. É interessante que essa associação se verificou apesar de a ICP ter sido realizada, nesses casos, sempre após período de estabilização do quadro clínico, durante vários dias. Mas também é oportuno frisar que, pela análise multivariada, o antecedente de SIMIs não foi considerado preditor independente de reestenose. Os resultados da presente investigação, associando calibre vascular e maior risco de RC com pequeno calibre vascular, também são particularmente coerentes com os descritos por Süsselbeck et al. ${ }^{29}$, que evidenciaram, em avaliação de 293 lesões, ser o D.L.R. e a presença de DM preditores de reestenose. Nesse estudo, foi descrito que, em vasos $<3,0 \mathrm{~mm}$, a presença de DM aumenta em duas vezes o risco de reestenose.

O volume de stent como preditor de reestenose, isolado, ou associado ao calibre do vaso tratado, já havia sido descrito por Hoffman et al. ${ }^{30}$, em estudo no qual foi utilizado o stent de Palmaz Schatz ${ }^{\text {TM }}$ (Cordis - Johnson \& Johnson). Vale salientar que esse conceito genérico fica amplamente corroborado pelo presente estudo, no qual foram utilizados vários tipos de stents, podendo seus resultados serem extrapolados para o mundo real, com maior confiabilidade.

Estes resultados, no entanto, contrastam com os descritos em estudo meta-analítico que avaliou população composta em três registros (MUSIC, WEST-II e ESSEX) e dois estudos multicêntricos (ERASER e TRAPIST), o qual concluiu que, em vasos relativamente grandes, a taxa de reestenose poderia ser predita pela mínima área de stent (inversamente relacionada) e pelo comprimento do stent (diretamente relacionada), mas não pelo volume do stent. Esta meta-análise visou à construção de tabela de referência para cálculo da taxa esperada de reestenose, a partir dos resultados finais da análise ultra-sonográfica quantitativa, mas tais observações derivaram de ICP realizada em vasos mais calibrosos e com lesões mais curtas do que no presente estudo.

Deve ser mencionado que o calibre do vaso tratado expresso angiograficamente pelo D.L.R. interfere na taxa de reestenose, mesmo após o advento dos stents farmacológicos. No estudo SIRIUS ${ }^{31}$, enquanto a taxa geral de reestenose no segmento do stent foi de $36,3 \%$ versus $8,9 \%$ (stent convencional versus farmacológico), ao avaliar-se o subgrupo de pacientes com vasos menores (tercil inferior), essas taxas de reestenose passaram para $42,9 \%$ e $18,4 \%$, respectivamente.

No presente estudo, não se focalizou o impacto clínico da RC. Entretanto, Elezi et al. ${ }^{32}$ também encontraram resultados semelhantes em série com mais de 2000 pacientes, na qual os pacientes com vasos de calibre $<2,8 \mathrm{~mm}$ apresentaram maior taxa de reestenose pelo critério binário, quando comparados àqueles com calibre superior a 3,2 mm (38,6 versus $20,4 \%)$. Nessa investigação, a maior taxa de reestenose esteve diretamente relacionada à evolução clínica, uma vez que naqueles pacientes com vasos menores, a sobrevida livre de eventos cardiovasculares graves em um ano foi de $69,5 \%$, versus $77,5 \%$ e $81 \%$, respectivamente para vasos com calibres intermediários e calibres maiores. Assim, a relevância clínica dos resultados do presente

TABELA 5

Análise multivariada dos preditores de reestenose angiográfica

\begin{tabular}{lccccc}
\hline Variável & Coeficiente Beta & Erro Padrão & Wald Coef./Ep & Prob. & Exp (Beta) \\
\hline D.L.M. & $-0,94573$ & 1,60857 & $-0,587947$ & 0,55 & 0,3884 \\
Volume stent & 4,34938 & 1,76364 & 2,46615 & $\mathbf{0 , 0 1 3}$ & 77,43 \\
Área stent & 2,19828 & 1,87928 & 1,16975 & 0,24 & 9,01 \\
D.L.R. & $-0,85389$ & 1,6749 & $-0,05982$ & 0,96 & 0,9182 \\
Constante & $-5,07521$ & 2,4083 & $-2,10738$ & 0,017 & - \\
\hline
\end{tabular}


Lima-Filho MO, et al. Volume do Stent à Ultra-Sonografia Intracoronária como Preditor de Reestenose Angiográfica. Estudo em Pacientes com Alto Risco de Reestenose. Rev Bras Cardiol Invas 2007; 15(2): 125-133.

trabalho de investigação torna-se realçada, com a confluência de dois fatores eminentemente clínicos e constitucionais - a disglicidemia e o reduzido calibre vascular - mostrando-se esta associação particular e potencialmente prejudicial para pacientes tratados com ICP.

\section{CONCLUSÃO}

Com base nestes resultados, conclui-se que, em população de pacientes consecutivos, nos quais foi realizada busca sistemática de alterações do metabolismo da glicose como provável indicador de maior risco de reestenose coronária, o calibre do vaso esteve relacionado de forma inversa à taxa de reestenose. Entre os parâmetros que incorporam essa característica, o volume de stent pelo USIC < 119,8 $\mathrm{mm}^{3}$ mostrouse preditor independente de reestenose coronária.

\section{REFERÊNCIAS BIBLIOGRÁFICAS}

1. Kuntz RE, Gibson CM, Nobuyoshi M, Baim DS. Generalized model of restenosis after conventional balloon angioplasty, stenting and directional atherectomy. J Am Coll Cardiol. 1993;21(1):15-25.

2. Silber S, Albertsson P, Aviles FF, Camici PG, Colombo A, Hamm C, et al. Guidelines for percutaneous coronary interventions. The Task Force for Percutaneous Coronary Interventions of the European Society of Cardiology. Eur Heart J. $2005 ; 26(8): 804-47$.

3. Kuntz RE, Baim DS. Defining coronary restenosis: newer clinical and angiographic paradigms. Circulation. 1993;88(3): 1310-23.

4. Topol EJ, Nissen SE. Our preoccupation with coronary luminology. The dissociation between clinical and angiographic findings in ischemic heart disease. Circulation. 1995;92(8):2333-42.

5. Smith SC Jr, Feldman TE, Hirshfeld JW Jr, Jacobs AK, Kern MJ, King SB $3^{\text {rd }}$, et al. ACC/AHA/SCAI 2005 Guideline Update for Percutaneous Coronary Intervention-Summary Article: A Report of the American College of Cardiology/ American Heart Association Task Force on Practice Guidelines (ACC/AHA/SCAI Writing Committee to Update the 2001 Guidelines for Percutaneous Coronary Intervention). J Am Coll Cardiol. 2006;47(1):216-35.

6. Hirshfeld JW Jr, Schwartz JS, Jugo R, MacDonald RG, Goldberg S, Savage MP, et al. Restenosis after coronary angioplasty: a multivariate statistical model to relate lesion and procedure variables to restenosis. The M-HEART Investigators. J Am Coll Cardiol. 1991;18(3):647-56.

7. Komatsu R, Ueda M, Naruko T, Kojima A, Becker AE. Neointimal tissue response at sites of coronary stenting in humans: macroscopic, histological, and immunohistochemical analyses. Circulation. 1998;98(3):224-33.

8. Akiyama T, Moussa I, Reimers B, Ferraro M, Kobayashi $\mathrm{Y}$, Blengino $S$, et al. Angiographic and clinical outcome following coronary stenting of small vessels: a comparison with coronary stenting of large vessels. J Am Coll Cardiol. 1998;32 (6):1610-8.

9. Mercado N, Boersma E, Wijns W, Gersh BJ, Morillo CA, de Valk $V$, et al. Clinical and quantitative coronary angiographic predictors of coronary restenosis: a comparative analysis from the balloon-to-stent era. J Am Coll Cardiol. 2001;38(3):645-52.

10. de Jaegere $P$, Mudra $H$, Figulla $H$, Almagor $Y$, Doucet $S$, Penn I, et al. Intravascular ultrasound-guided optimized stent deployment. immediate and 6 months clinical and angiographic results from the Multicenter Ultrasound Stenting in Coronaries Study (MUSIC study). Eur Heart J. 1998;19(8):1214-23.

11. de Feyter PJ, Kay P, Disco C, Serruys PW. Reference chart derived from post-stent-implantation intravascular ultrasound predictors of 6-month expected restenosis on quantitative coronary angiography. Circulation. 1999;100(17):1777-83.

12. White CJ, Ramee SR, Collins TJ, Escobar AE, Karsan A Shaw D, et al. Coronary thrombi increase PTCA risk. Angioscopy as a clinical tool. Circulation. 1996;93(2):253-8.

13. Violaris AG, Melkert R, Herrman JP, Serruys PW. Role of angiographically identifiable thrombus on long-term luminal renarrowing after coronary angioplasty: a quantitative angiographic analysis. Circulation. 1996;93(5):889-97.

14. Bauters C, Lablanche JM, McFadden EP, Hamon M, Bertrand ME. Relation of coronary angioscopic findings at coronary angioplasty to angiographic restenosis. Circulation. 1995;92 (9):2473-9.

15. Takagi T, Akasaka T, Yamamuro A, Honda Y, Hozumi T, Morioka S, et al. Troglitazone reduces neointimal tissue proliferation after coronary stent implantation in patients with non-insulin dependent diabetes mellitus: a serial intravascular ultrasound study. J Am Coll Cardiol. 2000;36(5): 1529-35.

16. Kasaoka S, Tobis JM, Akiyama T, Reimers B, Di Mario C, Wong ND, et al. Angiographic and intravascular ultrasound predictors of in-stent restenosis. J Am Coll Cardiol. 1998;32(6): 1630-5

17. Takagi T, Yoshida K, Akasaka T, Kaji S, Kawamoto $T$, Honda $Y$, et al. Hyperinsulinemia during oral glucose tolerance test is associated with increased neointimal tissue proliferation after coronary stent implantation in nondiabetic patients: a serial intravascular ultrasound study. J Am Coll Cardiol. 2000;36(3):731-8.

18. World Health Organization. Definition, diagnosis and classification of diabetes mellitus and its complications: part 1: report of a WHO consultation: diagnosis and classification of diabetes mellitus. Geneva, Switzerland:World Health Organization;1999.

19. Sirnes PA, Myreng Y, Molstad P, Golf S. Reproducibility of quantitative coronary analysis. Assessment of variability due to frame selection, different observers, and different cinefilmless laboratories. Int J Card Imaging. 1996;12(3):197-203.

20. Keane D, Haase J, Slager CJ, Montauban van Swijndregt E, Lehmann KG, Ozaki Y, et al. Comparative validation of quantitative coronary angiography systems. Results and implications from a multicenter study using a standardized approach. Circulation. 1995;91(8):2174-83.

21. van der Zwet PM, Reiber JH. A new approach for the quantification of complex lesion morphology: the gradient field transform; basic principles and validation results. J Am Coll Cardiol. 1994;24(1):216-24.

22. Reiber JH, Koning G, Goedhart B. The effect of DICOM on QCA and clinical trials. Int J Card Imaging. 1998;14(Suppl 1):7-12.

23. Dussaillant GR, Mintz GS, Pichard AD, Kent KM, Satler LF, Popma JJ, et al. Small stent size and intimal hyperplasia contribute to restenosis: a volumetric intravascular ultrasound analysis. J Am Coll Cardiol. 1995;26(3):720-4.

24. Muhlestein JB, Anderson JL, Horne BD, Lavasani F, Allen Maycock CA, Bair TL, et al. Effect of fasting glucose levels on mortality rate in patients with and without diabetes mellitus and coronary artery disease undergoing percutaneous coronary intervention. Am Heart J. 2003;146(2):351-8.

25. Cutlip DE, Chauhan MS, Baim DS, Ho KKL, Popma JJ, Carrozza JP, et al. Clinical restenosis after coronary stenting: 
Lima-Filho MO, et al. Volume do Stent à Ultra-Sonografia Intracoronária como Preditor de Reestenose Angiográfica. Estudo em Pacientes com Alto Risco de Reestenose. Rev Bras Cardiol Invas 2007; 15(2): 125-133.

perspectives from multicenter clinical trials. J Am Coll Cardiol. 2002;40(12):2082-9.

26. Bauters C, Hubert E, Prat A, Bougrimi K, Van Belle E, McFadden EP, et al. Predictors of restenosis after coronary stent implantation. J Am Coll Cardiol. 1998,31(6):1291-8.

27. Serryus PW, Kay IP, Disco C, Deshpande NV, de Feyter PJ. Periprocedural quantitative coronary angiography after PalmazSchatz stent implantation predicts the restenosis rate at six months: results of a meta-analysis of the BElgian NEtherlands Stent study (BENESTENT) I, BENESTENT II Pilot, BENESTENT II and MUSIC trials. Multicenter Ultrasound Stent In Coronaries. J Am Coll Cardiol. 1999;34(4):1067-74.

28. Kastrati A Schomig A, Elezi S, Schuhlen H, Dirschinger J, Hadamitzky $M$, et al. Predictive factors of restenosis after coronary stent placement. J Am Coll Cardiol. 1997;30(6):1428-36.
29. Suselbeck T, Latsch A, Siri H, Gonska B, Poerner T, Pfleger $S$, et al. Role of vessel size as a predictor for the occurrence of in-stent restenosis in patients with diabetes mellitus. Am J Cardiol. 2001;88(3):243-7.

30. Hoffmann R, Mintz GS, Dussaillant GR, Popma JJ, Pichard $A D$, Satler LF, et al. Patterns and mechanisms of in-stent restenosis. A serial intravascular ultrasound study. Circulation. 1996;94(6):1247-54.

31. Moses JW, Leon MB, Popma JJ, Fitzgerald PJ, Holmes DR, O'Shaughnessy C, et al. Sirolimus-eluting stens versus standard stents in patients with stenosis in a native coronary artery. N Engl J Med. 2003;349(14):1315-23.

32. Elezi S, Kastrati A, Neumann FJ, Hadamitzky M, Dirschinger J, Schomig A. Vessel size and long-term outcome after coronary stent placement. Circulation. 1998;98(18):1875-80. 